\title{
Renal Tubular Acidosis in Patients with Systemic Lupus Erythematosus
}

\author{
Necmi Eren ${ }^{a} \quad$ Ozkan Gungor $^{b} \quad$ Feyza Nur Sarisik ${ }^{b} \quad$ Fatih Sokmen $^{b}$ \\ Didem Tutuncu $^{b}$ Gozde Yildirim Cetin ${ }^{b}$ Ayten Yazicic ${ }^{c}$ Sibel Gökçay Bek ${ }^{a}$ \\ Eda Altun ${ }^{d}$ Orcun Altunoren $^{b}$ Ayse Cefle ${ }^{c}$ \\ a Department of Nephrology, Medical Faculty, Kocaeli University, Kocaeli, Turkey; \\ ${ }^{b}$ Department of Nephrology, Medical Faculty, Kahramanmaraş Sutcu Imam University, \\ Kahramanmaras, Turkey; ' ${ }^{\circ}$ Department of Rheumatology, Medical Faculty, Kocaeli University, \\ Kocaeli, Turkey; ${ }^{d}$ Department of Nephrology, Gölcük State Hospital, Kocaeli, Turkey
}

\section{Keywords}

Glomerular filtration rate · Proteinuria $\cdot$ Renal tubular acidosis · Systemic lupus erythematosus

\begin{abstract}
Objective: Renal tubular acidosis (RTA) is a clinical manifestation that occurs with insufficiency in restoring bicarbonate or disruption in hydrogen ion elimination as a result of a disruption in tubulus functions, causing normal anion gap-opening metabolic acidosis. In the present study, we aimed to investigate the prevalence of RTA in the largest systemic lupus erythematosus (SLE) patient population to date. Materials and Methods: SLE patients, who were followed up in 2 different healthcare centers, were included. Patients with metabolic acidosis ( $\mathrm{pH}<7.35$ and $\mathrm{HCO}_{3}<22 \mathrm{mEq} / \mathrm{L}$ ) in venous blood gas analysis were determined. The serum and urine anion GAP of these patients were estimated, and the urine $\mathrm{pH}$ was assessed. RTA presence was evaluated as metabolic acidosis with a normal serum anion gap and a positive urine anion GAP. Results: A total of 108 patients were included in the present study. The mean age of the patients was $41.5 \pm 1.2$ and $87 \%$ were female. The SLE diagnosis duration was $75 \pm 5$ months. The mean creatinine value was $0.6 \pm 0.1 \mathrm{mg} / \mathrm{dL}$ and the mean eGFR was $111 \pm$ $2 \mathrm{~mL} / \mathrm{min}$. According to the blood gas analysis, 18 patients (16.7\% of the total) had RTA. Sixteen of these patients had type 1 RTA and 2 had type 2 RTA; type 4 RTA was not determined in any of the patients. Conclusion: RTA should be considered in SLE patients even if they have normal eGFR values. This is the largest study to examine the prevalence of RTA in SLE patients in the literature.

\section{Karger'}




\section{Introduction}

Systemic lupus erythematosus (SLE) is a multisystem autoimmune disease with a broad spectrum of clinical presentations encompassing almost all organs and tissues. The extreme heterogeneity of the disease has caused some investigators to propose that SLE represents a syndrome rather than a single disease. The prevalence rates in lupus are estimated to be as high as 51 per 100,000 people in the USA [1]. The incidence of lupus increased nearly 3 -fold in the last 40 years [1]. Sixty-five percent of patients with SLE have a disease onset between the ages of 16 and 55 years.

Renal involvement occurs in 40-70\% of all SLE patients and it is a major cause of morbidity and hospital admissions [2]. Immune complex formation/deposition in the kidney leads to intraglomerular inflammation with activation and proliferation of leukocytes [3]. Urinalysis is the most important and effective method to detect and monitor renal activity of disease. Clinical findings such as microscopic hematuria, microalbuminuria, asymptomatic proteinuria, massive proteinuria and nephrotic syndrome, rapidly progressive glomerulonephritis, and acute and chronic renal failure can be seen [4].

Renal tubular acidosis (RTA) is defined as an ion transport disorder in renal tubules. Four types are named and described as follows: distal or type 1 RTA, i.e., a defect in hydrogen ion excretion from the kidneys; proximal or type $2 \mathrm{RTA}$, i.e., a defect in bicarbonate $\left(\mathrm{HCO}_{3}\right)$ reabsorption; mixed or type 3 RTA, i.e., a defect involving both of these; and type 4 RTA, i.e., aldosterone deficiency or resistance [5]. RTA may develop due to idiopathic, familial and secondary causes. Diabetes mellitus, rheumatoid arthritis, Sjögren syndrome, lysosomal storage diseases and drugs are among the common causes of secondary RTA [6-8]. It is known that RTA can be seen in patients with SLE [9]. Studies in the literature are generally in the form of case reports. Although the mechanism of RTA formation in SLE is not clear, different mechanisms have been reported.

Although some studies with small cohorts and several case reports exist in the literature, to the best of our knowledge our study is the largest one to examine the prevalence of RTA in SLE patients.

\section{Materials and Methods}

Patients followed-up with the diagnosis of SLE in 2 nephrology and rheumatology units were included in this cross-sectional study. The diagnosis of SLE was based on the diagnostic criteria revised by the American College of Rheumatology in 1997 [10].

Patients with a glomerular filtration rate (GFR) $<60 \mathrm{~mL} / \mathrm{min}$ were not included in this study due to the metabolic acidosis effect of renal failure. In addition, patients diagnosed with diabetes mellitus or Sjögren syndrome and those treated with diuretics, drugs affecting the potassium metabolism, and sodium hydrogen carbonate therapy were not included in this study.

The demographic data of the patients (age, sex, disease duration, additional diseases, medications, renal biopsy findings, autoantibody and complement levels, and 24-h urine protein quantity) were obtained from the patient files. All blood samples were obtained from patients in the morning after $12 \mathrm{~h}$ of fasting. Biochemical tests in serum (urea, creatinine, sodium, potassium, and chlorine) and urine (sodium, potassium, chlorine, etc.) were studied using the enzymatic colorimetric technique (ADVIA ${ }^{\circledR} 1800$ Clinical Chemistry System; Siemens, Erlangen, Germany). An ABL800 (Radiometer Medical ApS; Copenhagen, Denmark) device was used for blood gas analysis. All urine analyses were performed on H-800 and FUS-200 automatic modular urine analyzers (Dirui Industry, Changchun, China).

Urine $\mathrm{pH}$, venous blood gas analysis parameters including serum $\mathrm{pH}$ values, the $\mathrm{HCO}_{3}$ concentration, base excess, the presence of metabolic acidosis, which was defined as low $\mathrm{HCO}_{3}(<22 \mathrm{mEq} / \mathrm{L})$ together with low serum $\mathrm{pH}$ values $(<7.35)$, were checked at the last follow-up. Patients with metabolic acidosis were further analyzed for urine anion gap (UAG) and serum anion gap (SAG) using the following formulas: UAG =

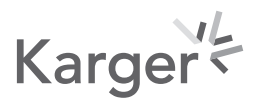


Kidney and

Blood Pressure

Research

Table 1. Summary of demographic and laboratory data

\begin{tabular}{l|l}
\hline Kidney Blood Press Res 2020;45:883-889 \\
\hline DOI: 10.1159/000509841 & $\begin{array}{l}\text { ○ 2020 The Author(s). Published by S. Karger AG, Basel } \\
\text { www.karger.com/kbr }\end{array}$ \\
\hline
\end{tabular}

Value

Age, years

Male/female ratio

Duration of disease, months

Hypertension, \%

BUN, mg/dL

Creatinine, $\mathrm{mg} / \mathrm{dL}$

Potassium, mmol/L

Uric acid, $\mathrm{mg} / \mathrm{dL}$

Albumin, g/dL

Hemoglobin, g/dL

CRP, mg/L

Proteinuria, mg/day

eGFR, $\mathrm{mL} / \mathrm{min}$

Patients with antinuclear antibody positivity, $n$

Patients with anti-dsDNA positivity, $n$

Patients with low C3 levels, $n$

Kidney biopsy results, $n$

Type 2 lupus nephritis

Type 3 lupus nephritis

Type 4 lupus nephritis

Type 5 lupus nephritis

Renal tubular acidosis, \%
$41.5 \pm 1.2$

$14 / 94$

$75 \pm 5$

30.7

$20 \pm 1$

$0.6 \pm 0.1$

$4.2 \pm 0.4$

$4.4 \pm 1.3$

$4.1 \pm 0.1$

$12.2 \pm 0.1$

$2.9 \pm 0.3$

$339 \pm 68$

$111 \pm 2$

77

28

23

9

3

19

5

16.7

Values are presented as means \pm SD.

$\left[\mathrm{Na}^{+}\right]+\left[\mathrm{K}^{+}\right]-\left[\mathrm{Cl}^{-}\right]$and SAG $=\left[\mathrm{Na}^{+}\right]-[\mathrm{Cl}]+\mathrm{HCO}$. SAG values between 7 and 14 were accepted as normal. RTA was defined as metabolic acidosis with normal SAG and positive UAG values. Patients with RTA were further categorized as having type 1 distal RTA if the urine $\mathrm{pH}$ was $>5.5$ and the serum potassium level was low or normal; they were categorized as having type 2 RTA if the urine $\mathrm{pH}$ was $<5.5$ and the serum potassium level was low or normal, and they were considered to have type 4 RTA if the serum potassium level was $>5.5$ $\mathrm{mEq} / \mathrm{L}[11]$.

\section{Statistical Analysis}

Continuous variables were tested for a normal distribution using Kolmogorov-Smirnov and ShapiroWilk tests. We report continuous data as means \pm SD. Median values were used when a normal distribution was absent. We compared parametric continuous variables using the t test, and the Mann-Whitney U test was used for nonparametric continuous variables. Categorical variables were summarized as percentages and compared with the $\chi^{2}$ test. $p<0.05$ was considered statistically significant, and 95\% CI were set. All statistical analyses were performed using SPSS version 15 (SPSS, Inc., Chicago, IL, USA).

\section{Results}

One hundred eight patients were included in this study. The mean age of the patients was $41.5 \pm 1.2$ and $87 \%$ of them were female. The mean duration of SLE disease was $75 \pm 5$ months, and $30.7 \%$ of patients were hypertensive. There was biopsy-proven SLE nephritis in 36 patients (33\%), and the majority of them had class 4 lupus nephritis (55\%). Sixty-nine percent of the patients were taking mycophenolate mofetil and a low-dose steroid. The mean creatinine level was $0.6 \pm 0.1 \mathrm{mg} / \mathrm{dL}$ and the mean eGFR level was $111 \pm 2 \mathrm{~mL} / \mathrm{min}$. A summary of the demographic and laboratory data is shown in Table 1.

\section{Karger'k}


Table 2. Comparison of the data in 2 groups involving patients with and without RTA

\begin{tabular}{lccc}
\hline & $\begin{array}{c}\text { RTA }(+) \\
(n=18)\end{array}$ & $\begin{array}{c}\text { RTA }(-) \\
(n=90)\end{array}$ & $p$ value \\
\hline Age, years & $45.1 \pm 2.4$ & $40.8 \pm 1.3$ & 0.13 \\
Duration of disease, months & $73.4 \pm 13.9$ & $75.8 \pm 5.7$ & 0.66 \\
Hypertension, \% & 44.4 & 27.8 & 0.13 \\
BUN, mg/dL & $21.2 \pm 4.1$ & $20.5 \pm 1.1$ & 0.47 \\
Creatinine, mg/dL & $0.6 \pm 0.1$ & $0.6 \pm 0.1$ & 0.28 \\
Albumin, g/dL & $4.1 \pm 0.1$ & $4.1 \pm 0.1$ & 0.86 \\
Hemoglobin, g/dL & $12.1 \pm 0.3$ & $12.2 \pm 0.1$ & 0.86 \\
CRP, mg/L & $2.5 \pm 0.5$ & $2.9 \pm 0.4$ & 0.64 \\
Proteinuria, mg/day & $558 \pm 268$ & $301 \pm 65$ & 0.36 \\
eGFR, mL/min & $98 \pm 5$ & $114 \pm 2$ & $\mathbf{0 . 0 1}$ \\
Serum sodium, mEq/L & $141 \pm 3$ & $140 \pm 11$ & 0.94 \\
Urine sodium, mEq/L & $88 \pm 45$ & $95 \pm 56$ & 0.59 \\
Serum chlorine, mEq/L & $107 \pm 4$ & $107 \pm 8$ & 0.33 \\
Urine chlorine, mEq/L & $112 \pm 57$ & $121 \pm 66$ & 0.65 \\
Serum potassium, mEq/L & $4.4 \pm 0.1$ & $4.3 \pm 0.1$ & 0.13 \\
Urine potassium, mEq/L & 30.9 & 46.9 & 0.44 \\
Serum PH & $7.31 \pm 0.29$ & $7.37 \pm 0.04$ & $<\mathbf{0 . 0 0 1}$ \\
Urine PH & 6 & 5.9 & 0.26 \\
Serum anion gap & $11.3 \pm 2.0$ & $13.3 \pm 3.7$ & $\mathbf{0 . 0 3 3}$ \\
Urine anion gap & 17 & 0.4 & $\mathbf{0 . 0 3}$ \\
\hline
\end{tabular}

Values are presented as means $\pm \mathrm{SD}$.

According to the blood gas analysis, 18 patients (16.7\%) had RTA. The level of serum anion gap was $11.3 \pm 2$, and the urine anion gap was 17 (10-29.8) in patients with RTA. Sixteen of those patients had type 1 RTA and 2 had type 2 RTA. Type 4 RTA was not seen in any patient. The mean age of the patients with RTA was $45.1 \pm 2.4$ years and the mean disease duration of those patients was $73.4 \pm 13.9$ months. There were 10 patients diagnosed with SLE nephritis by biopsy, and those patients were taking mycophenolate mofetil. When the patients with and without an RTA diagnosis were compared, there were no differences in terms of age, sex, duration of disease, treatment, or complement and autoantibody levels. Although the proteinuria amount of the patients with RTA was higher than in those without RTA, this difference was not found to be statistically significant. Although the eGFR levels of both groups were quite good, the eGFR levels of the patients with RTA were significantly lower. The serum $\mathrm{pH}$ and $\mathrm{HCO}_{3}$ levels in the RTA group were significantly lower than those in the non-RTA group. There were no statistical differences between the 2 groups in terms of serum creatinine, serum and urine chlorine (107 \pm 4 vs. $107 \pm 8$ and $112 \pm 57$ vs. $121 \pm 66$ $\mathrm{mEq} / \mathrm{L}$, respectively), serum and urine sodium (141 \pm 3 vs. $140 \pm 11$ and $88 \pm 45$ vs. $95 \pm 56$ $\mathrm{mEq} / \mathrm{L}$, respectively), urine potassium levels (30.9 [22.5-64.8] vs. $46.9 \mathrm{mEq} / \mathrm{L}$ [25.4-75.4]) and urine $\mathrm{pH}(6[6-6.5]$ vs. 5.9 [5.5-6.5]) $(p>0.05)$. A comparison of the data in 2 groups involving patients with and without RTA is shown in Table 2.

\section{Discussion}

The presence of RTA in patients with SLE has been previously reported in case reports. In this cross-sectional study, we aimed to investigate the incidence of RTA in a large population of SLE patients, and we detected that the incidence was remarkable at a rate of $16.7 \%$. 
SLE is a multisystem autoimmune disease with an unknown etiology. In the course of the disease, it is not wrong to say that the kidney is the organ that affects mortality and morbidity. The kidneys are among the most important organs during active disease [12]. The rates of renal involvement during the course of SLE are very variable and have been reported to be $25-60 \%$ in adults and $30-80 \%$ in children [13]. It was suggested that this ratio may be as high as $75-100 \%$ by biopsy. The severity of the disease varies from silent kidney disease to endstage renal failure. In patients with urinary proteinuria, the presence of erythrocyte cylinders, detection of hematuria, pyuria, an increase in serum creatinine, a decrease in serum complement-3 (C3) and complement-4 (C4) levels, a decrease in creatinine clearance, and hypertension should suggest renal involvement [14]. Patients with renal involvement should have an early diagnosis and treatment should be initiated. In $10-30 \%$ of those patients, endstage renal failure occurs despite appropriate treatment. The 15-year survival rate has increased to $80 \%$ with new treatment methods [15].

RTA refers to a group of disorders characterized by a defective renal acid-base regulation. The normal urine acidification capacity is impaired and results in a net acid retention and hyperchloremic metabolic acidosis. Acid-base imbalance occurs despite a normal or mildly reduced GFR. RTA is classified into 4 main forms, i.e., distal, proximal, mixed, and hyperkalemic RTA. Distal RTA is associated with a reduced urine acid secretion, proximal RTA is characterized by impaired $\mathrm{HCO}_{3}$ reabsorption, and hyperkalemic RTA is an acid-base disorder generated by aldosterone deficiency or resistance [5]. Type 1, 2, 4 RTA cases have been reported as accompanying SLE, in the literature, and type 1 RTA has been detected in the majority of these cases. In our study, type 1 RTA was seen in 16 out of 18 patients with RTA. The development of type 4 RTA in SLE is extremely rare, though there are several case reports in the literature [16]. Aldosterone deficiency and/or resistance is responsible for its mechanism. None of our patients had type 4 RTA.

Electrolyte and acid-base disorders are key components of each disorder. RTA is often detected incidentally after laboratory tests, but some patients present with clinical features such as poor growth, dehydration, or an altered mental state [5]. RTA may develop due to idiopathic, familial, and secondary causes. Diabetes mellitus, rheumatoid arthritis, Sjögren syndrome, sarcoidosis, storage diseases (such as cystinosis and Wilson disease), and drugs (lithium, amphotericin b, and trimethoprim) are among the secondary causes $[17,18]$. SLE is also among the secondary causes of RTA. In some studies, it has been reported that SLE constitutes $12 \%$ of distal RTA causes [19].

There may be glomerular, tubular, or interstitial involvement of the kidneys in SLE patients. In particular, tubular and interstitial involvement may lead to RTA and/or hyperkalemia.

The only study investigating tubular defects in SLE was performed by Kozeny et al. [20]. Kozeny et al. [20] found distal RTA in 8 (26\%) out of 30 SLE patients undergoing a renal biopsy. In that study, no relation was detected between glomerular findings and the presence of RTA. In our study, there was biopsy-proven SLE nephritis in 10 out of 18 patients diagnosed with RTA. It should be kept in mind that, RTA may be present synchronously in patients with lupus nephritis. The pathogenesis of RTA development in SLE is unknown. RTA is also seen in autoimmune diseases such as RA and Sjögren syndrome, and it is thought that hypergammaglobulinemia and immunological factors may be responsible for the pathogenesis in RTA [20]. Again, it is thought that, antibodies against the carbonic anhydrase enzyme, or tubulointerstitial inflammation may have a role [20]. It has been suggested that a high disease activity score may be a risk factor for RTA development in SLE patients [21], but supporting studies are needed. In our study, when SLE patients with and without an RTA diagnosis were compared, there were no differences in terms of age, sex, duration of disease, or autoantibody and complement levels. The proteinuria amounts of the patients with RTA were higher (statis-

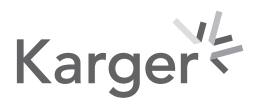


tically insignificant); however, it was shown that most of the patients in this group had a previous glomerular involvement. Although, the GFR values of both groups were in the normal range, there was a significant difference between the GFR levels in the 2 groups and the GFR level was lower in group with an RTA diagnosis. It has been suggested that glomerular function has a minimal or no effect on the development of RTA. In the study conducted by Kocyigit et al. [22] and our previous study [11] on renal transplant patients, it was shown that the GFR has an effect on RTA development. Supporting studies on this subject are also needed.

Our study has some limitations. Blood gas analysis and serum and urine anion gap measurements were performed one time. Since kidney biopsy is invasive and its absolute indications did not exist in all of the patients, the severity of glomerular and tubular damage could not evaluated by kidney biopsy in all of the patients. Another limitation is that we may not have been able to identify all patients who may have incomplete distal RTA. An ammonium chloride $\left(\mathrm{NH}_{4} \mathrm{Cl}\right)$ loading test is useful in such cases. These patients may not have a $\mathrm{pH}$ level below 7.35 or a decrease in $\mathrm{HCO}_{3}$ in their serum, and metabolic acidosis can be induced by applying $\mathrm{NH}_{4} \mathrm{CI}(0.1 \mathrm{~g} / \mathrm{kg}$ for 3 days). Under these stimulated acidemia conditions, if the urine $\mathrm{pH}$ level is above 5.3, it indicates distal RTA.

In conclusion, we showed that the incidence of RTA was not negligible in a large proportion of the SLE patients, whose GFR levels were within the normal ranges. We think that the evaluation of SLE patients, especially those with metabolic acidosis or potassium imbalance in this respect, would be useful, and supporting studies on this subject are needed.

\section{Statement of Ethics}

The study protocol was performed in accordance with the Declaration of Helsinki. The Ethics Committee of the Medical Faculty of Kahramanmaraş Sutcu Imam University approved the study protocol (KSU KAEK: 2018/31). Written informed consent was obtained from all subjects before enrollment into this study.

\section{Conflict of Interest Statement}

The authors declare that they have no conflict of interests.

\section{Funding Sources}

This study was not funded by any organization.

\section{Author Contributions}

Data collection, data analysis: F.N.S., F.S., D.T., G.Y.C., A.Y., S.G.B., E.A., and A.C. Data collection, data analysis, conception or design, authorship: N.E., O.G., and O.A. 


\section{References}

1 Rees F, Doherty M, Grainge M, Davenport G, Lanyon P, Zhang W. The incidence and prevalence of systemic lupus erythematosus in the UK, 1999-2012. Ann Rheum Dis. 2016 Jan;75(1):136-41.

2 Tang Y, Zhang X, Ji L, Mi X, Liu F, Yang L, et al. Clinicopathological and outcome analysis of adult lupus nephritis patients in China. Int Urol Nephrol. 2015 Mar;47(3):513-20.

3 Hui M, Garner R, Rees F, Bavakunji R, Daniel P, Varughese S, et al. Lupus nephritis: a 15-year multi-centre experience in the UK. Lupus. 2013 Mar;22(3):328-32.

4 Yap DY, Yung S, Chan TM. Lupus nephritis: an update on treatments and pathogenesis. Nephrology (Carlton). 2018 Oct;23 Suppl 4:80-3.

5 Sharma S, Gupta A, Saxena S. Comprehensive clinical approach to renal tubular acidosis. Clin Exp Nephrol. 2015 Aug;19(4):556-61.

6 Bello CH, Duarte JS, Vasconcelos C. Diabetes mellitus and hyperkalemic renal tubular acidosis: case reports and literature review. J Bras Nefrol. 2017 Oct-Dec;39(4):481-5.

7 Varshney AN, Prasad P, Kumar N, Singh NK. Acute-onset paralysis in a patient of rheumatoid arthritis. Ann Saudi Med. 2015 Jan-Feb;35(1):69-71.

8 Jung SW, Park EJ, Kim JS, Lee TW, Ihm CG, Lee SH, et al. Renal Tubular Acidosis in Patients with Primary Sjögren's Syndrome. Electrolyte Blood Press. 2017 Sep;15(1):17-22.

9 Li SL, Liou LB, Fang JT, Tsai WP. Symptomatic renal tubular acidosis (RTA) in patients with systemic lupus erythematosus: an analysis of six cases with new association of type 4 RTA. Rheumatology (Oxford). 2005 Sep; 44(9):1176-80.

10 Marc C, Hochberg MD. MPH. Updating the American College of Rheumatology revised criteria for the classification of systemic lupus erythematosus. Arthritis Rheum. 1997 Sep;40(9):1725.

11 Tanrisev M, Gungor O, Kocyigit I, Kurtulmus Y, Tugmen C, Colak H, et al. Renal tubular acidosis in renal transplant patients: the effect of immunosuppressive drugs. Ann Transplant. 2015 Feb;20:85-91.

12 Wen YK. Renal biopsy findings in new-onset systemic lupus erythematosus with clinical renal disease. Int Urol Nephrol. 2011 Sep;43(3):801-6.

13 Saxena R, Mahajan T, Mohan C. Lupus nephritis: current update. Arthritis Res Ther. 2011;13(5):240.

14 Singh S, Saxena R, Palmer BF. Lupus nephritis. Am J Med Sci. 2009 Jun;337(6):451-60.

15 Zhang X, Ji L, Yang L, Tang X, Qin W. The effect of calcineurin inhibitors in the induction and maintenance treatment of lupus nephritis: a systematic review and meta-analysis. Int Urol Nephrol. 2016 May;48(5):73143.

16 Nalcacioglu H, Genc G, Meydan BC, Ozkaya O. Hyperkalaemia in a female patient with systemic lupus erythematosus. Pediatr Nephrol. 2012 Sep;27(9):1499-503.

17 Patel MC. Trimethoprim related Hyperkalaemia in a patient with Renal Tubular Acidosis Type 4. Acute Med. 2009;8(3):117-8.

18 Hemstreet BA. Antimicrobial-associated renal tubular acidosis. Ann Pharmacother. 2004 Jun;38(6):1031-8.

19 Both T, Zietse R, Hoorn EJ, van Hagen PM, Dalm VA, van Laar JA, et al. Everything you need to know about distal renal tubular acidosis in autoimmune disease. Rheumatol Int. 2014 Aug;34(8):1037-45.

20 Kozeny GA, Barr W, Bansal VK, Vertuno LL, Fresco R, Robinson J, et al. Occurrence of renal tubular dysfunction in lupus nephritis. Arch Intern Med. 1987 May;147(5):891-5.

21 Akin D, Ozmen S. Type 4 renal tubular acidosis in a patient with lupus nephritis. Iran J Kidney Dis. 2014 Jan; 8(1):73-5.

22 Kocyigit I, Unal A, Kavuncuoglu F, Sipahioglu MH, Tokgoz B, Oymak O, et al. Renal tubular acidosis in renal transplantation recipients. Ren Fail. 2010 Jul;32(6):687-90. 